Recebido em 11/2019. Aceito para publicação em 01/2020.

\title{
SÍNTESE DE MATERIAL NANOESTRUTURADO À BASE DE RESINA EPOXÍDICA E SÍLICA: ESTUDO DAS PROPRIEDADES TERMOMECÂNICAS
}

\section{SYNTHESIS OF NANOSTRUCTURED MATERIAL BASED ON EPOXY RESIN AND SILICA: STUDY OF THE THERMOMECHANICAL PROPERTIES}

\author{
Patricia Reis Pinto 1 \\ Washington Fagundes de Jesus ${ }^{2}$ \\ Adriana dos Anjos da Silva ${ }^{3}$ \\ Jonathan da Silva Santana ${ }^{4}$
}

Resumo: A ciência e tecnologia em nanoescala têm atraído considerável atenção nos últimos anos, pela expectativa do impacto que os materiais nanoestruturados podem causar na melhoria da qualidade de vida. Espera-se que o avanço da nanociência e da nanotecnologia estimule a exploração de novos fenômenos e novas teorias. Neste trabalho foram investigados os principais aspectos envolvidos na modificação da resina epóxi (RE), curada com agente endurecedor poliamida. Nesta matriz epoxídica, incorporados nanopartículas inorgânicas de sílica comercial - R200 (Aerosil). A caracterização das amostras dos sistemas epóxi/poliamida, com e sem adição de nanocarga de sílica foram avaliadas por Termogravimetria (TG), Termogravimetria derivativa (DTG) e análise dinâmica mecânica (DMA). A reometria das amostras constatou que pela determinação do regime de viscoelasticidade linear da matriz epoxídica, verificou-se que a adição de $1 \% \mathrm{~m} / \mathrm{m}$ da sílica resultou no aumento da viscosidade da matriz epoxídica, como esperado. Este comportamento sugere que a dispersão resultou na maior interação entre a ER e a carga de sílica, promovido pelo alto cisalhamento durante o procedimento de mistura.

Palavras-chave: Epóxi; poliamida; sílica; propriedades termomecânicas; material nanoestruturado.

Abstract: Science and technology at the nanoscale have attracted considerable attention in recent years, because of the expected impact that the nanostructured materials can have on the improvement of life quality. It is expected that the advance in nanoscience and nanotechnology encourages the exploration of new phenomena and new theories. The present study investigated the main aspects involved in the modified of epoxy resin (RE) cured with hardener polyamide agent. In this epoxy matrix are incorporated inorganic nanoparticles of commercial silica - R200 (AerosilTM). The characterization of the samples from the systems epoxy / polyamide, with and without the addition of nanoclay silica, were evaluated by thermogravimetry (TG), derivative thermogravimetry (DTG) and dynamic mechanical analysis (DMA). The rheometry of the samples indicated that, by the determination of the linear viscoelasticity regime of the epoxy matrix, it was verified that the addition of $1 \% \mathrm{w} / \mathrm{w}$ of silica resulted in increased viscosity of the epoxy matrix, as expected. This behavior suggests that the dispersion resulted in increased interaction between the RE and silica filler, promoted by high shear during the mixing procedure.

Keywords: Epoxy; polyamide; silica; thermomechanical properties; nanostructured material.

\footnotetext{
1 Fundação Centro Universitário Estadual da Zona Oeste, UEZO, Brasil. E-mail: patriciarpinto@yahoo.com.br.

2 Fundação Centro Universitário Estadual da Zona Oeste, UEZO, Brasil. E-mail: cinematica7@gmail.com.

3 Fundação Centro Universitário Estadual da Zona Oeste, UEZO, Brasil. E-mail: anjosadriana@eq.ufrj.br.

4 Fundação Centro Universitário Estadual da Zona Oeste, UEZO, Brasil. E-mail: jsantana.uezo@gmail.com.
} 


\section{INTRODUÇÃO}

O termo nanocompósitos foi introduzido por pesquisadores, entre 1982-1983, para designar materiais compósitos contendo mais de uma fase sólida. Os nanocompósitos são materiais bifásicos, em que há uma interpenetração das fases em uma escala nanométrica. As propriedades desses materiais não são descritas apenas pela soma das contribuições individuais de cada fase, este efeito vai para além do somatório de propriedades, pois existe um sinergismo que depende também da natureza química dos segmentos orgânicos e inorgânicos e do tamanho e morfologia dos correspondentes domínios (FERREIRA; RANGEL, 2009; CARVALHO, 2015; REZENDE; BOTELHO, 2000).

Pode-se encontrar na literatura vários exemplos de materiais com propriedades superiores, que são afetadas pelo tamanho de partícula. Essas modificações são conhecidas como efeitos quânticos de tamanho quando a carga (na faixa de 1-10 nm) está confinada na fase amorfa, podem acontecer no caso da argila, alumina e sílica, em matriz epóxi (MACIEL; LONGO; LEITE, 2003). O conhecimento das propriedades dos materiais nanoestruturados, relacionadas à sua dimensão, é muito importante como para o desenvolvimento de futuras aplicações tecnológicas (ROENNER et al., 2017; AHMED et al.; KAYA; YILMAZ; DEVECI, 2018; PAVOSKI et al., 2018; SALIMIAN et al., 2018; LI et al., 2018; GAO et al., 2018; SINGH et al., 2019; HAZARIKA; KARAK, 2019; KARNATI; AGBO; ZHANG, 2020).

Existem basicamente três principais técnicas utilizadas na obtenção do nanocompósitos poliméricos, são elas; intercalação das nanocargas intercalação no polímero fundido, a técnica por meio da polimerização in situ a mistura de soluções contendo nanocargas com subsequente evaporação do solvente. Uma combinação de dois ou mais métodos também pode ser utilizada (COELHO, 2008). No método de intercalação as nanocargas são acrescidas ao polímero durante o processamento deste, neste caso as nanocargas podem ou não passar por um tratamento prévio. $\mathrm{Na}$ polimerização in situ, a nanocarga é dispersa em um monômero ou uma solução deste, para que o polímero formado durante a reação de polimerização incorpore as nanocargas dispersas no meio reacional. E o método em solução consiste em usar um solvente que além de dissolver polímero também disperse a nanocarga. A mistura das soluções com a subsequente retirada do solvente dará origem ao nanocompósito. (ESTEVES; TIMMOS; TRINDADE, 2004; ANADÃO; WIEBECK; VALENZUELA-DÍAZ, 2011).

O presente trabalho procurou dispersar as nanopartículas da sílica comercial R200 (Aerosil@) em um dos pré-polímeros - na fração epoxídica - para posteriormente promover a reação de polimerização com o outro pré-polímero e endurecedor poliamida e avaliar as propriedades termomecânicas dos materiais nanoestruturados. 


\section{PROCEDIMENTO EXPERIMENTAL}

\subsection{Obtenção dos Compósitos}

A resina epóxi comercial adquirida para a proposta desse trabalho foi constituída por um sistema bicomponente; o pré polímero epoxidado $(E R)$ e o agente endurecedor - poliamida líquida reativa. As nanocargas de sílica foram acrescentadas a fração epoxídada (ER) na proporção de 5.0, 2.5, e 1.0\% m/m, respectivamente. A dispersão da nanocarga foi realizada utilizando homogeneizador Turrax a $17500 \mathrm{rpm}$, sob banho de aquecimento à temperatura de $50^{\circ} \mathrm{C}$ por $25 \mathrm{~min}$ visando aumentar a fluidez da matriz epoxídica. Posteriormente o sistema foi desgaseificado sob vácuo durante 10 minutos. $O$ agente endurecedor (poliamida) foi adicionado as frações epoxidadas (ER) na proporção de 80phr. Os dois componentes (ER com nanocargas e endurecedor) foram misturados por meio do dispersor Speed Mixer por 2 ciclos de $5 \mathrm{~min}$ a 3500rpm. Em seguida, a mistura foi vertida em moldes de silicone de dimensões apropriadas para os ensaios de caracterização. O ciclo de cura estabelecido para a reação de polimerização consistiu de duas etapas: a primeira de 24 horas a temperatura ambiente; seguida da segunda etapa de 3 horas a $150^{\circ} \mathrm{C}$.

\subsection{Caracterizações}

\subsubsection{Análise termogravimétrica (TGA)}

A análise termogravimétrica foi realizada em equipamento SDT Q600 TA Instruments. As análises termogravimétricas foram realizadas a uma taxa de aquecimento de $20^{\circ} \mathrm{C} / \mathrm{min}$, em atmosfera de nitrogênio. Os perfis da TGA foram registrados na faixa de temperatura entre 30 a $700^{\circ} \mathrm{C}$.

\subsubsection{Análise Dinâmica mecânica (DMA)}

Dentre as propriedades dinâmico-mecânicas, o módulo de armazenamento ( $\left.E^{\prime}\right)$, e a tan $\delta$ foram determinados para todos os sistemas epoxídicos. As medidas foram realizadas com o DMTA utilizando como garra do tipo flexão em dois pontos, com deformação fixada em $0,1 \%$, e $1 \mathrm{~Hz}$ de frequência. Os ensaios foram feitos em uma faixa de temperatura de 25 a $200^{\circ} \mathrm{C}$ e com taxa de aquecimento de $3^{\circ} \mathrm{C} / \mathrm{min}$.

\subsubsection{Reologia}

A reometria das dispersões ER/sílica foi monitorada através da viscosidade dinâmica complexa $\left(\eta^{*}\right)$ em regime oscilatório. Inicialmente, para conhecimento da região de viscoelasticidade linear da matriz de ER foi feito o estudo da varredura de deformação $(0,1-100 \%)$, a frequência de $1 \mathrm{~Hz}$ e temperatura ambiente. Posteriormente, a avaliação do grau de dispersão da sílica na matriz foi realizada pelo ensaio de varredura de frequência sob regime oscilatório, para determinação da viscosidade complexa $\left(\eta^{*}\right)$ das misturas não curadas. As análises reológicas foram executadas a temperatura ambiente, em uma faixa de frequência de $0,1-100 \mathrm{~Hz}, 1 \%$ de deformação, e a distância entre as placas paralelas (gap) de 500 $\mathrm{mm}$. 


\section{RESULTADOS E DISCUSSÃO}

\subsection{Análise termogravimétrica (TGA)}

A análise termogravimétrica é definida como um processo contínuo, que envolve a medida da variação de massa de uma amostra em função da temperatura ou do tempo. A amostra pode ser aquecida ou resfriada a uma velocidade selecionada, ou pode ser mantida a uma temperatura fixa (LUCAS; SOARES; MONTEIRO, 2001).

As amostras dos sistemas epóxi/poliamida, com e sem adição de nanocarga de sílica foram avaliadas por Termogravimetria (TG) e Termogravimetria derivativa (DTG). A Figura 1 apresenta o perfil da curva de TG para todas as amostras e para os sistemas puros. Tanto as amostras puras, sem nanocarga, quanto os nanocompósitos apresentaram semelhança quanto ao perfil da curva de termogravimetria. O perfil geral das curvas apresentou o início da temperatura de degradação em torno de $300^{\circ} \mathrm{C}$ e o final em torno de $515^{\circ} \mathrm{C}$. Em aproximadamente $790^{\circ} \mathrm{C}$ os eventos de perda de massa foram concluídos.

Verifica-se no gráfico de TG (Figura 1) que as amostras com 1\% de nanocarga representada pelas cores vermelhas (80phr) e amarela (54phr) apresentaram pequenos deslocamentos para menores valores de temperatura de degradação, quando comparado com os respectivos sistemas puros, sem adição de sílica. Pode ser que a quantidade de sílica nesses sistemas tenha promovido um efeito de distanciamento das cadeias poliméricas, diminuindo as interações intermoleculares entre as cadeias e influenciando a temperatura de degradação.

Figura 1 - TGA curva termogravimétrica.

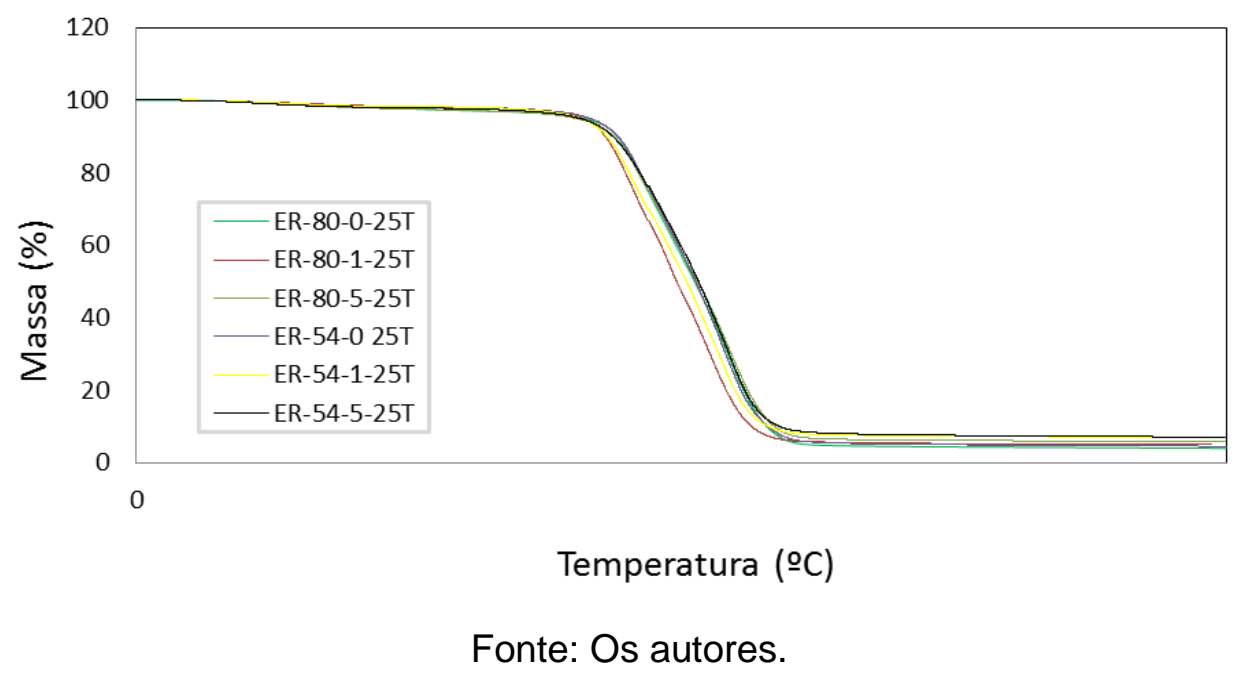

A Tabela 1 apresenta os valores das temperaturas do início da perda de massa, bem como do início e final da degradação da matriz polimérica. Também são apresentados os valores dos percentuais dos resíduos após término do evento. Verificase que com o aumento do teor da nanocarga de sílica aos nanocompósitos foi observado um aumento nos percentuais de resíduo conforme esperado. 
Tabela 1 - Temperatura de degradação dos nanocompósitos (TGA).

\begin{tabular}{rccccc}
\hline Amostra & Sílica $(\% \mathrm{~m} / \mathrm{m})$ & $\mathrm{T}$ inicial $\left({ }^{\circ} \mathrm{C}\right)$ & $\begin{array}{c}\mathrm{T} \text { decomp. } \\
\left({ }^{\circ} \mathrm{C}\right)\end{array}$ & $\mathrm{T}$ final $\left({ }^{\circ} \mathrm{C}\right)$ & $\begin{array}{c}\% \text { Resíduo } \\
\left(792^{\circ} \mathrm{C}\right)\end{array}$ \\
\hline 54-W0 & 0 & 18,54 & 300,37 & 500,28 & 4,551 \\
54-W1 & 1 & 26,67 & 307,92 & 507,02 & 6,995 \\
54-W2 & 5 & 21,37 & 307,36 & 514,64 & 7,098 \\
\hline 80-W3 & 0 & 20,46 & 309,41 & 493,29 & 4,014 \\
80-W4 & 1 & 31,60 & 317,07 & 494,71 & 4,983 \\
80-W5 & 5 & 21,15 & 304,11 & 493,33 & 5,852 \\
\hline
\end{tabular}

Fonte: Os autores.

Na termogravimetria derivada (DTG) as curvas são registradas a partir das curvas TGA e correspondem à derivada primeira da variação de massa em relação ao tempo $(d m / d T=f)$ ou em função da temperatura $(d m / d t=f)$. A curva DTG é muito útil nos casos em que o registro de TG apresentam sobreposições decorrentes da amostra, a DTG permite determinar a taxa de variação de massa e fornece facilmente informação adicional para encontrar $\mathrm{T}_{\text {onset }} \mathrm{e} \mathrm{T}_{\text {endset }}$ (CANEVAROLO; MATOS; MACHADO, 2007).

Embora as curvas de TG apresentem aparentemente um único perfil de decaimento, observa-se pelas curvas de DTG dos materiais obtidos que há pelo menos 3 eventos de perda de massa preponderantes, conforme podem serem observados nas Figuras de 2 a 5 . A tabela 2 apresenta os valores de temperatura para a taxa máxima de degradação $\left(T_{1}, T_{2}, T_{3}\right.$ e $T_{1}{ }^{\prime}, T_{2}{ }^{\prime}$ e $\left.T_{3}{ }^{\prime}\right)$ dos eventos térmicos principais visualizados nos gráficos de DTG para os nanocompósitos de RE com e sem adição de sílica.

Tabela 2 - Valores da temperatura para a taxa máxima de degradação das curvas de DTG.

\begin{tabular}{ccccc}
\hline Amostra & Sílica $(\% \mathrm{~m} / \mathrm{m})$ & $1^{\circ}$ Evento $\left({ }^{\circ} \mathrm{C}\right)$ & $2^{\circ}$ Evento $\left({ }^{\circ} \mathrm{C}\right)$ & $3^{\circ}$ Evento $\left({ }^{\circ} \mathrm{C}\right)$ \\
54-W0 & 0 & 375 & 418 & 427 \\
54-W1 & 1 & 362 & 410 & 426 \\
54-W2 & 5 & 374 & 412 & 432 \\
80-W3 & 0 & 375 & - & 432 \\
80-W4 & 1 & 360 & 388 & 419 \\
80-W5 & 5 & 374 & 393 & 432 \\
\hline
\end{tabular}

Fonte: Os autores.

A Figura 2 apresenta a comparação entre a curva de DTG para a amostra com e sem acréscimo de $1 \%$ de nanocarga de sílica à concentração de 54 phr de poliamida. Observa-se que os perfis das curvas de DTG são semelhantes, apresentando três eventos principais na faixa de temperatura compreendido entre 362 a $427^{\circ} \mathrm{C}$. Verificase que o acréscimo de $1 \%$ de nanocargas de sílica promoveu pequena diminuição dos 
valores de temperatura de degradação do material levando a temperatura de $375^{\circ} \mathrm{C}(\mathrm{T} 1)$ para $362^{\circ} \mathrm{C}$ (T1') para o primeiro evento de degradação. No segundo evento a temperatura de degradação foi bastante semelhante $418^{\circ} \mathrm{C}$ (T2) e $410^{\circ} \mathrm{C}$ (T2'). E no terceiro evento a temperatura de degradação também foi semelhante $427^{\circ} \mathrm{C}$ (T3) e $426^{\circ} \mathrm{C}\left(\mathrm{T} 3^{\prime}\right)$.

Figura 2 - Comparação entre a curva termogravimétrica derivada 54 phr na RE com acréscimo de $\mathrm{SiO} 2$ 0,0\% e 1,0\%.

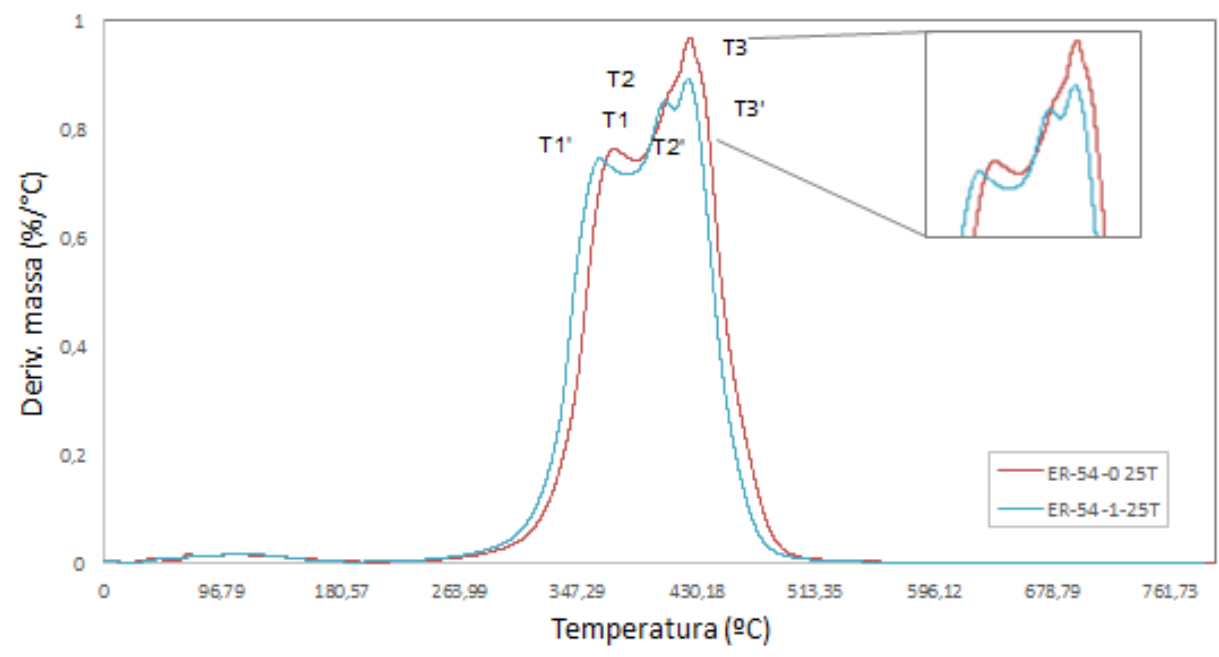

Fonte: Os autores.

Figura 3 - Comparação entre a curva termogravimétrica derivada 54 phr na RE com acréscimo de $\mathrm{SiO} 2$ 0,0\% e 5,0\%.

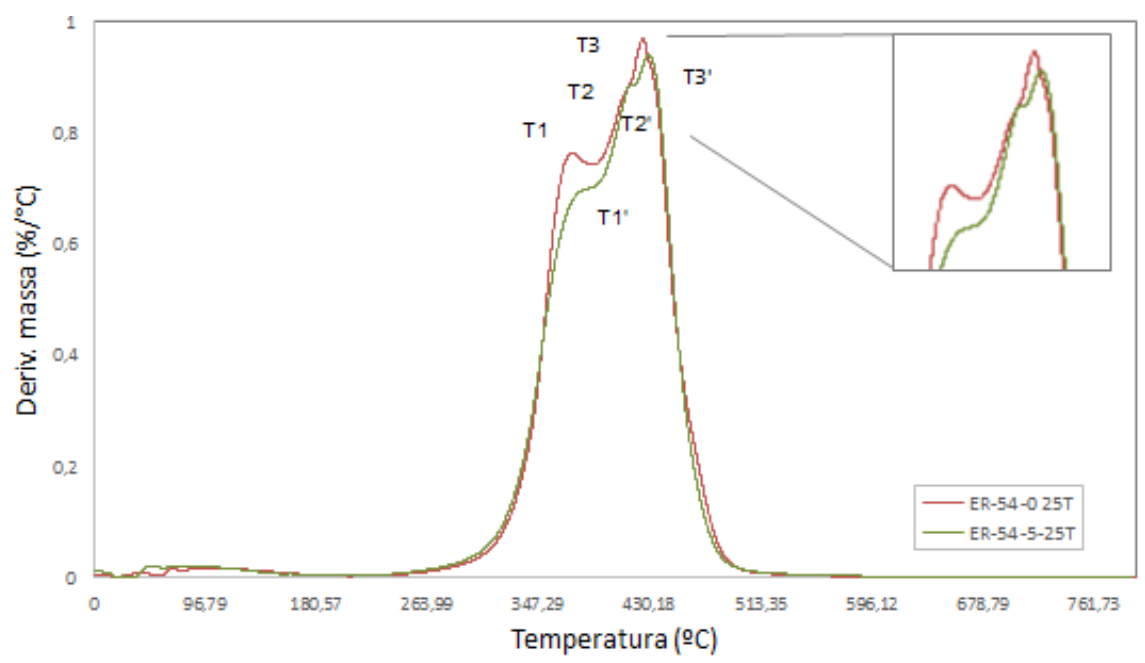

Fonte: Os autores.

Quando o acréscimo de nanocargas de sílica é de $5 \%$, para a mesma concentração de poliamida (54phr), o perfil das curvas de DTG, com e sem adição de nanocarga, se assemelham ainda mais, entre si. As temperaturas de degradação para os três eventos principais não sofrem variações consideráveis, conforme mostra a figura 
3. No primeiro evento térmico, os valores de temperatura de degradação do material variaram de $375^{\circ} \mathrm{C}(\mathrm{T} 1)$ para $374^{\circ} \mathrm{C}\left(\mathrm{T} 1^{\prime}\right)$. No segundo evento a temperatura de degradação foi de $418^{\circ} \mathrm{C}$ (T2) e $412^{\circ} \mathrm{C}$ (T2'). E no terceiro evento a temperatura de degradação também foi semelhante $427^{\circ} \mathrm{C}$ (T3) e $432^{\circ} \mathrm{C}$ (T3').

A Figura 4 apresenta a comparação entre a curva de DTG para a amostra com e sem acréscimo de $1 \%$ de nanocarga de sílica à concentração de 80 phr de poliamida. Observa-se que os perfis das curvas de DTG são diferentes. A curva de DTG para a amostra sem acréscimo de nanocarga apresenta seis eventos de degradação, enquanto que a curva de DTG para a amostra com acréscimo de 1\% de nanocarga de sílica apresenta três eventos principais na faixa de temperatura compreendido entre 360 a $432^{\circ} \mathrm{C}$. A presença da nanocarga de sílica alterou o perfil de degradação do material. Verifica-se que o acréscimo de $1 \%$ de nanocargas de sílica promoveu pequena diminuição dos valores de temperatura de degradação do material levando a temperatura de $375^{\circ} \mathrm{C}(\mathrm{T} 1)$ para $360^{\circ} \mathrm{C}\left(\mathrm{T} 1^{\prime}\right)$ para o primeiro evento de degradação. $\mathrm{O}$ segundo evento (T2), está em uma região de ruído e, portanto, não é possível identificar o pico da temperatura. A temperatura de decomposição encontrada para o segundo evento foi $388^{\circ} \mathrm{C}\left(\mathrm{T} 2^{\prime}\right)$, o menor valor investigado nesta caracterização. Para o terceiro evento foi observado um decréscimo da temperatura $432^{\circ} \mathrm{C}$ (T3) para $419^{\circ} \mathrm{C}$ (T3').

Figura 4 - Comparação entre a curva termogravimétrica derivada 80 phr na RE com acréscimo de SiO2 0,0\% e 1,0\%.

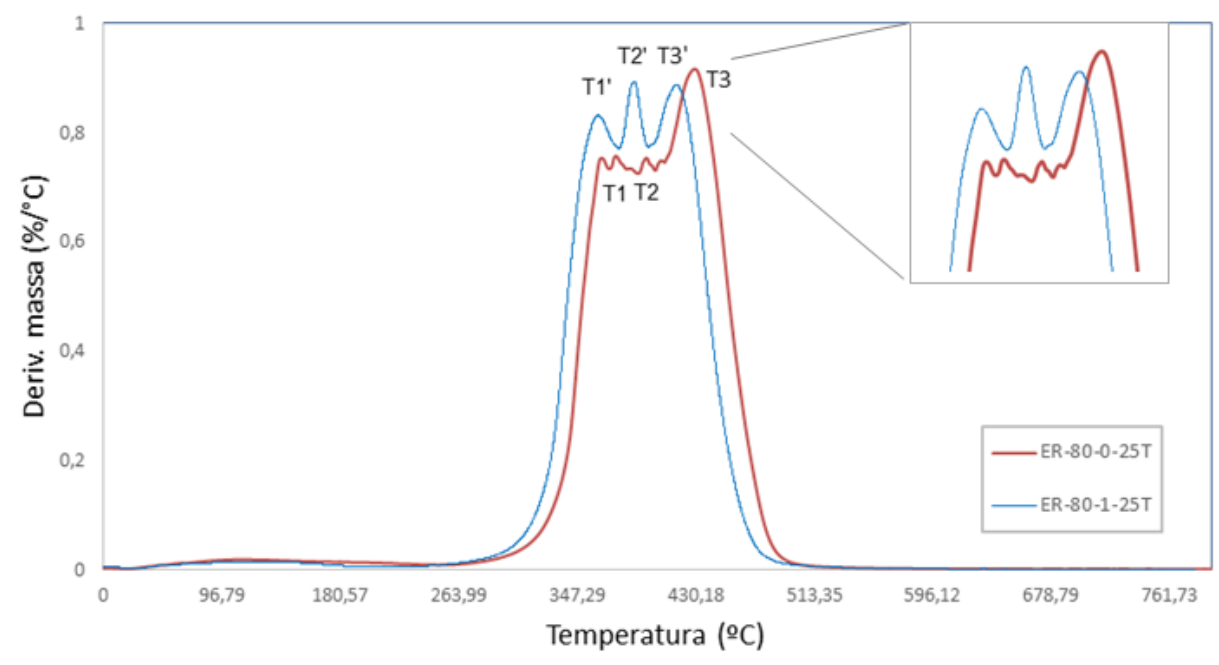

Fonte: Os autores.

A Figura 5 apresenta a comparação entre a curva de DTG para a amostra com e sem acréscimo de $5 \%$ de nanocarga de sílica à concentração de 80 phr de poliamida. Observa-se que os perfis das curvas de DTG se assemelham ainda mais, entre si. A curva de DTG para a amostra sem acréscimo de nanocarga apresenta cinco eventos de degradação, enquanto que a curva de DTG para a amostra com acréscimo de $5 \%$ de nanocarga de sílica apresenta três eventos principais na faixa de temperatura compreendido entre 375 a $432^{\circ} \mathrm{C}$. A presença da nanocarga de sílica alterou o perfil de degradação do material. Verifica-se que o acréscimo de $5 \%$ de nanocargas de sílica 
promoveu pequena diminuição dos valores de temperatura de degradação do material levando a temperatura de $375^{\circ} \mathrm{C}$ (T1) para $374^{\circ} \mathrm{C}$ (T1') para o primeiro evento de degradação. O segundo evento (T2), está em uma região de ruído e, portanto, não foi possível determinar o pico da temperatura. Para T2' a temperatura de decomposição encontrada foi correspondente a $393^{\circ} \mathrm{C}$. E no terceiro evento a temperatura de degradação também foi semelhante $432^{\circ} \mathrm{C}$ para (T3) e (T3').

Figura 5 - Comparação entre a curva termogravimétrica derivada 80 phr na RE com acréscimo de $\mathrm{SiO} 2$ 0,0\% e 5,0\%.

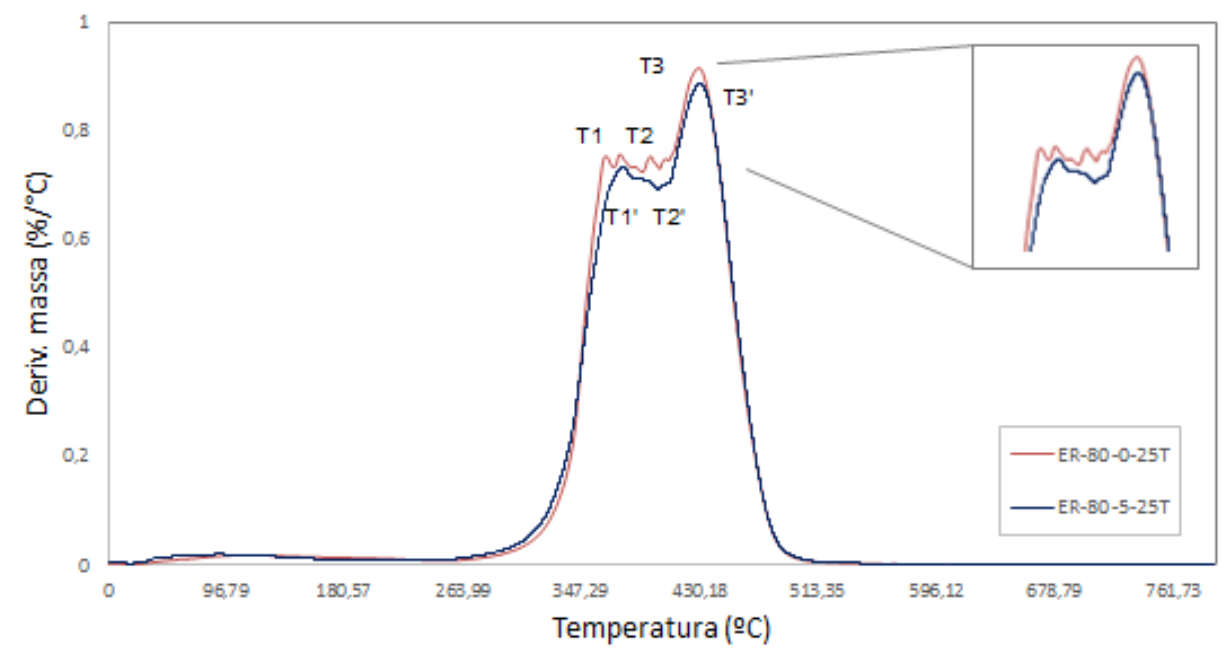

Fonte: Os autores.

\subsection{Análise Dinâmica Mecânica (DMA)}

Análise Dinâmico-Mecânica tem sido usada para caracterização de polímeros através da detecção dos processos de relaxação molecular. O DMA é capaz de fornecer informações a respeito do comportamento viscoelástico do sistema, desmembrando o módulo em duas componentes: a contribuição elástica e a viscosa. Essa técnica fornece informações a respeito do módulo de armazenamento ( $E$ '), do módulo de perda (E”) e da tangente de perda ou amortecimento mecânico (Tan $\delta=$ E'/E') de um material quando sujeito a uma solicitação dinâmica. A partir dessas variáveis, pode se investigar as propriedades como tenacidade, resistência ao impacto, resistência a propagação de trinca e rigidez entre outros. O valor máximo do pico da curva Tan $\delta$, foi critério escolhido para comparar os valores de $\mathrm{Tg}$ a partir da curva Tan $\delta$ versus temperatura, correspondendo à temperatura máxima do pico (CANEVAROLO, 2007; CARVALHO, 2015).

A tabela 3 mostra os resultados de DMA do sistema epóxi/poliamida sem carga (80-w0) e dos sistemas epóxi/poliamida obtidos com sílica (80-w1 e 80w-5). Pode-se observar que apenas o sistema sílica/poliamida com 1\% de sílica (80-w1) apresentou módulo de armazenamento (E') superior ao sistema epóxi/poliamida sem carga (80-w0). No entanto, todos os sistemas obtidos com adição de nanocarga apresentaram temperaturas de transição vítrea $(\mathrm{Tg})$ superiores ao sistema puro, sugerindo que houve 
uma boa interação da matriz epóxi/poliamida com a nanocarga de 239ílica.

Tabela 3 - Valores de Tg e módulo de armazenamento para as dispersões em sílica.

\begin{tabular}{cccc}
\hline Amostra & Silica $(\% \mathrm{~m} / \mathrm{m})$ & $\mathrm{E}^{\prime}(\mathrm{Mpa})$ & $\operatorname{tg}\left({ }^{\circ} \mathrm{C}\right)$ \\
$80-w 0$ & 0 & 1462 & 552 \\
$80-w 1$ & 1 & 1534 & 562 \\
$80-w 5$ & 5 & 1194 & 569 \\
\hline
\end{tabular}

Fonte: Os autores.

As figuras de 6 a 8 mostram as curvas do módulo de armazenamento ( $E$ ') para o sistema epóxi/poliamida puro e com adição de carga. Nota-se que a amostra com adição de $1 \%$ de carga apresentou o maior valor de módulo de armazenamento, conforme tabela 5 (1534Mpa) e não a amostra com $5 \%$ de nanocarga como era esperado. Isso pode ser atribuído a um efeito de dispersão. A matriz com menor teor de nanocarga tem a maior probabilidade de ser melhor distribuída na matriz polimérica. Tal efeito pode ter favorecido a maior interação matriz/nanocarga promovendo a redução na movimentação segmental das cadeias poliméricas refletindo no valor do módulo de armazenamento.

Figura 6 - Gráfico do Log Tan $\delta$ versus temperatura para amostra sem adição de nanocarga.

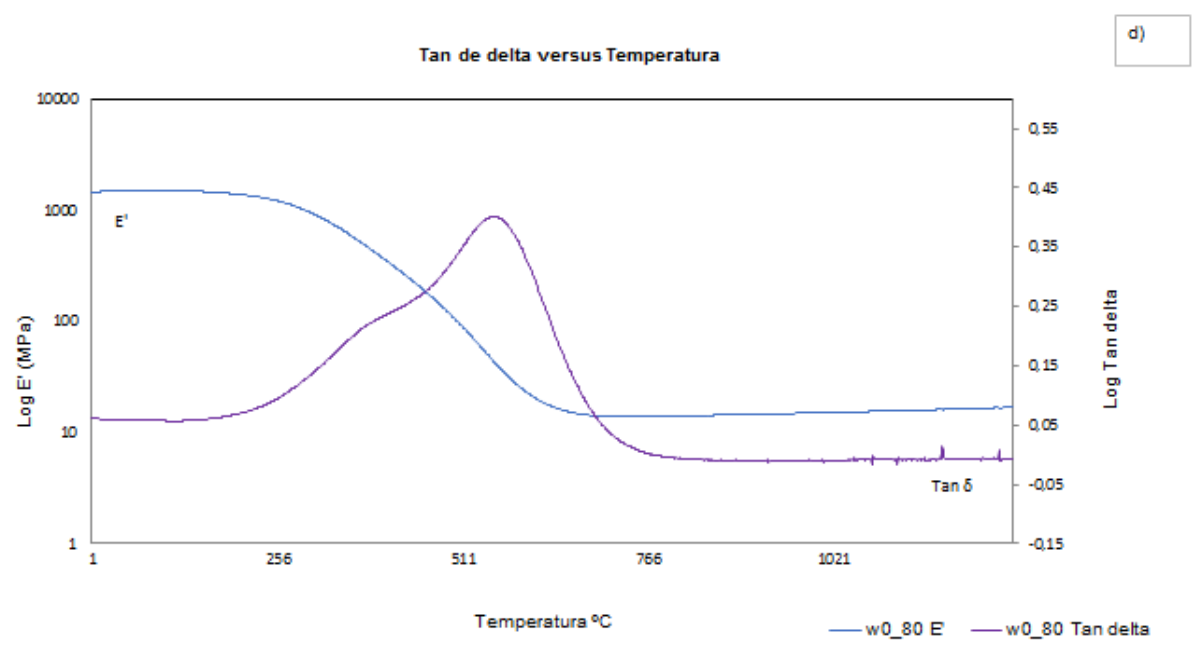

Fonte: Os autores. 
Figura 7 - Gráfico do Log do módulo de armazenamento versus temperatura para amostra sem adição de nanocarga.

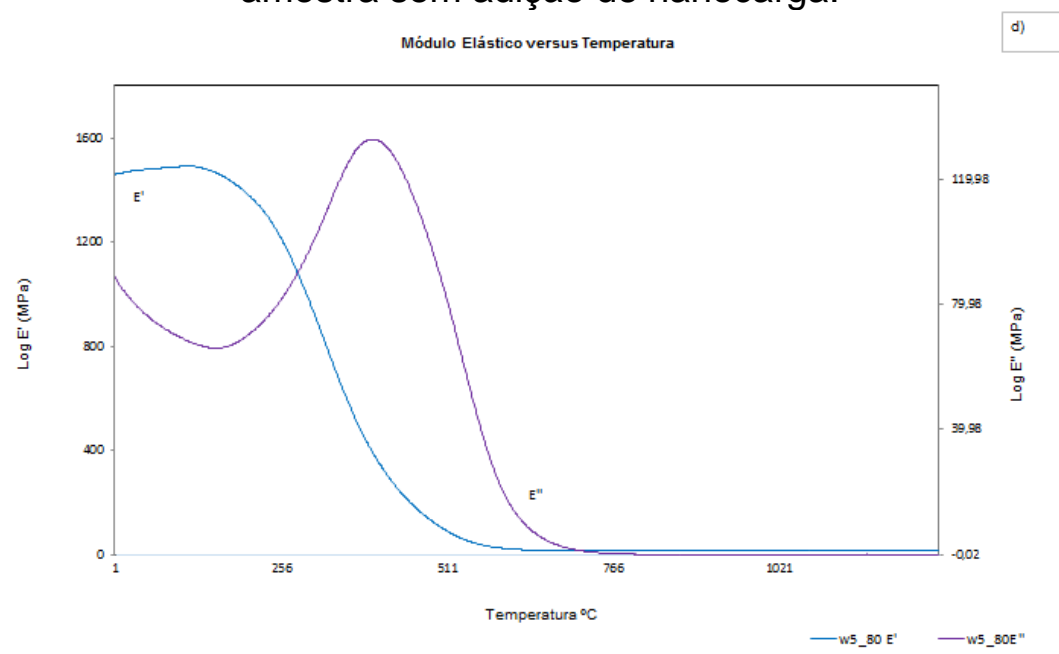

Fonte: Os autores.

Figura 8 -- Gráfico do Log Tan $\delta$ versus temperatura para amostra com $1 \%$ de nanocarga de sílica em epóxi.

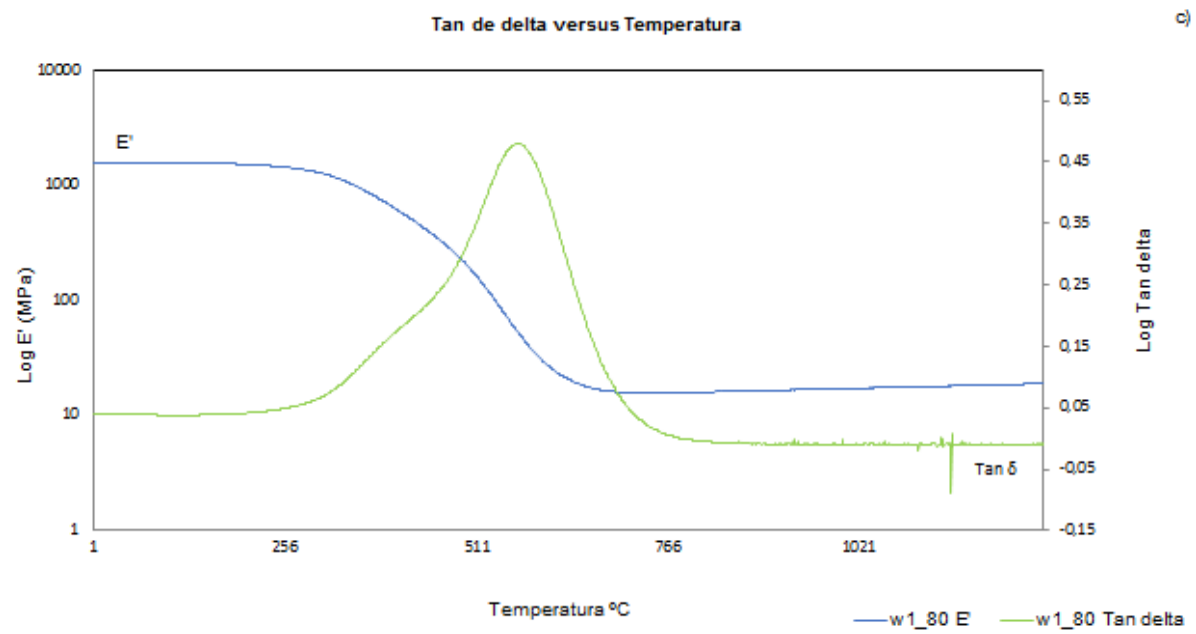

Fonte: Os autores. 
Figura 9 - Gráfico do Log do módulo de armazenamento versus temperatura para amostra com $1 \%$ de nanocarga de $\mathrm{SiO} 2$ em epóxi.

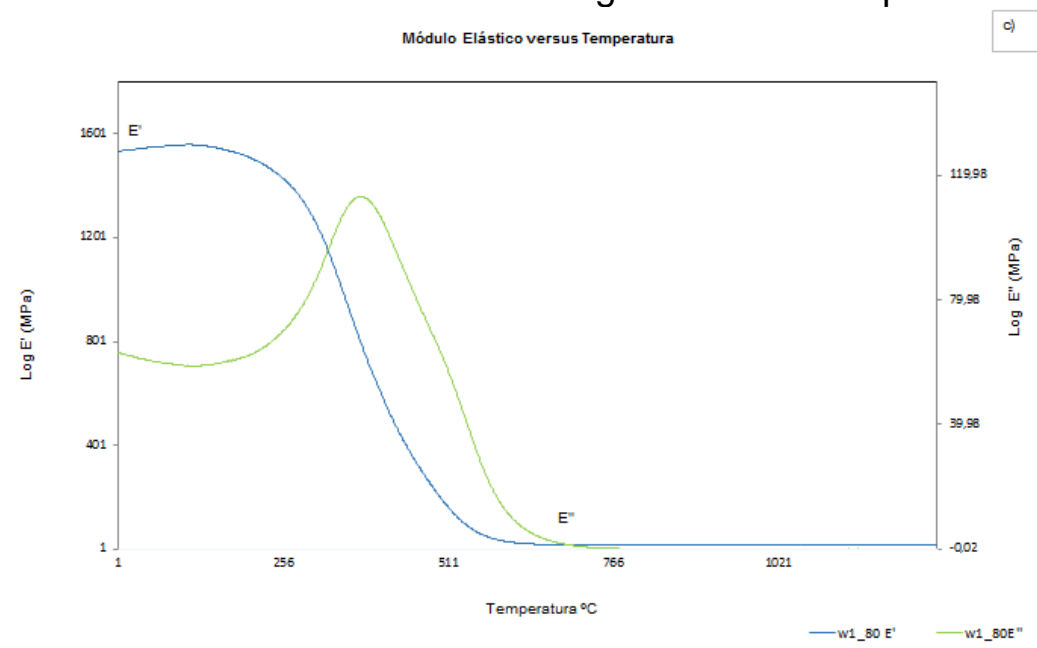

Fonte: Os autores.

Figura 10 - Gráfico do Log Tan $\delta$ versus temperatura para amostra com 5\% de nanocarga de sílica em epóxi.

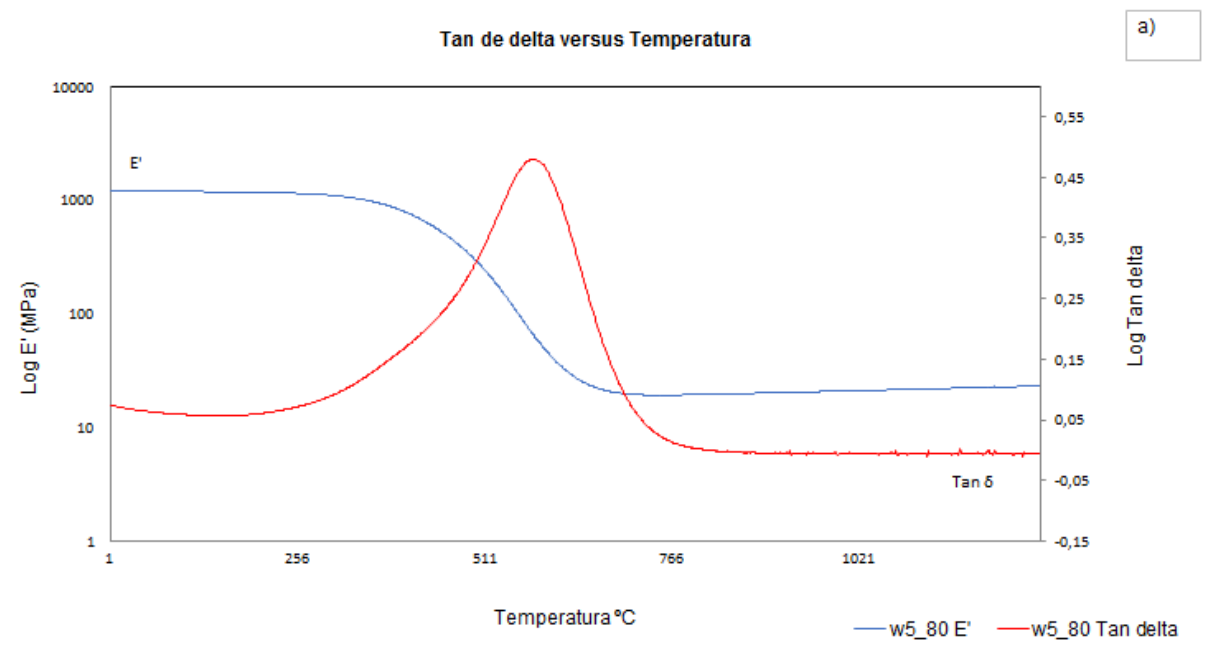

Fonte: Os autores. 
Figura 11 - Gráfico do Log do módulo de armazenamento versus temperatura para amostra com 5\% de nanocarga de $\mathrm{SiO} 2$ em epóxi.

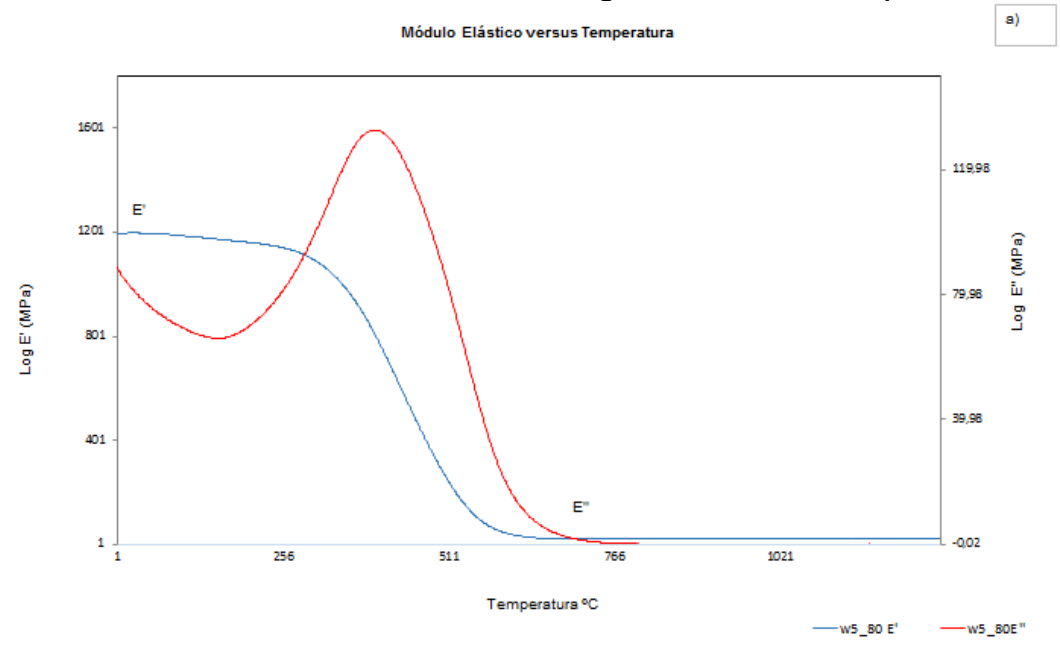

Fonte: Os autores.

\subsection{Análise Reológica}

\subsubsection{Estudo da dispersão}

A reometria das amostras iniciou pela determinação do regime de viscoelasticidade linear da matriz epoxídica. A Figura 12 mostra que em toda a faixa de deformação, a viscosidade da ER é constante, tal como o comportamento de um fluido newtoniano. Por outro lado, a adição da sílica não provocou alteração no perfil da viscosidade da matriz epoxídica. A Figura 12 mostrou que a viscosidade da amostra com $5 \% \mathrm{~mm}$ sílica apresenta comportamento linear em toda faixa de deformação.

Figura 12 - Viscosidade em função da deformação da resina epoxídica e amostra contendo $5 \% \mathrm{~m} / \mathrm{m}$ de sílica.

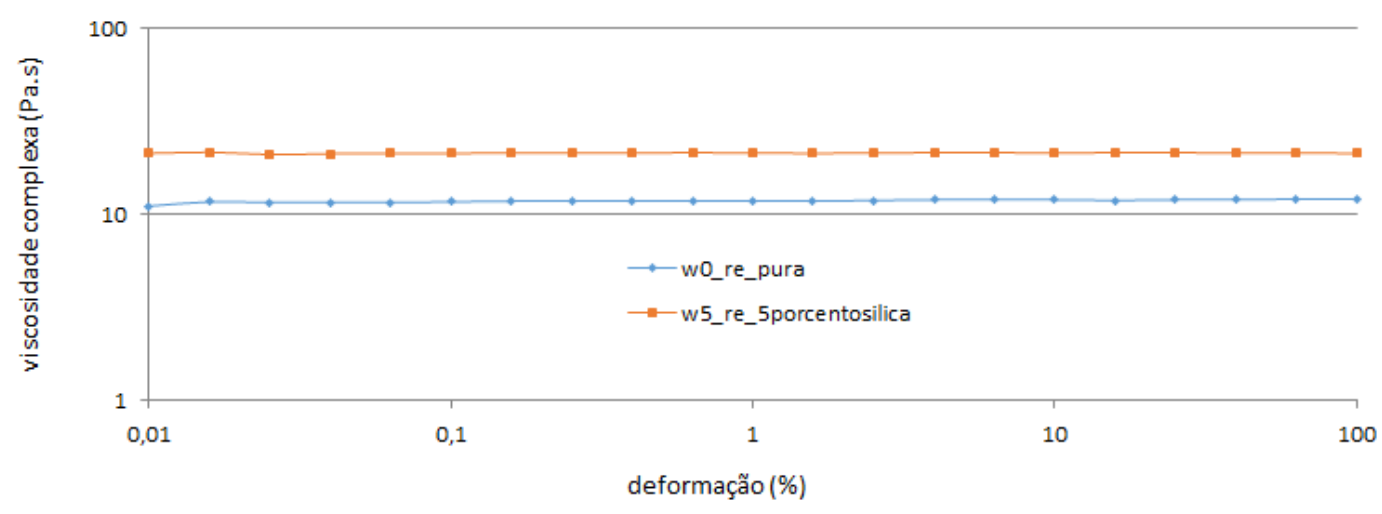

Fonte: Os autores.

A reometria também foi utilizada para avaliação do grau de dispersão da carga de sílica na matriz ER. A Figura 13 mostra que a adição de $1 \% \mathrm{~m} / \mathrm{m}$ da sílica resultou no aumento da viscosidade da matriz epoxídica, como esperado. Este comportamento sugere que a dispersão resultou na maior interação entre a ER e a carga de sílica, 
promovido pelo alto cisalhamento durante o procedimento de mistura. É conhecido que as nanocargas, como a sílica apresentam forte tendência a aglomeração em virtude da alta energia superficial das nanopartículas inorgânicas (CHEN et al, 2008; REDDY; PATRA; DAS, 2009). Dentre as estratégias para promover a desagregação e dispersão das nanocargas na matriz polimérica, destaca-se o alto cisalhamento obtido pelo uso de dispersores mecânicos na preparação das amostras. Desta forma, pode-se atribuir o aumento da viscosidade da mistura com a sílica à alta dispersão promovida pelo misturador Turrax. O alto cisalhamento promovido durante a dispersão da sílica na resina epoxídica, com o auxílio do misturador pode ter provocado a desagregação da nanocarga resultando no aumento da interação interfacial matriz/carga. Contudo, na Figura 13 é observado o aumento da viscosidade com o teor de sílica. A maior proporção da nanocarga de sílica resulta em maior resistência ao fluxo (viscosidade) devido a maior possibilidade de interação interfacial entre a resina epóxi e a sílica.

Figura 13 - Resistência ao fluxo das dispersões incorporadas com nanocarga SiO2, w0 - RE 0\%, w1 - RE 1\%, w2,5 - RE 2,5\% e w5 - RE 5\%.

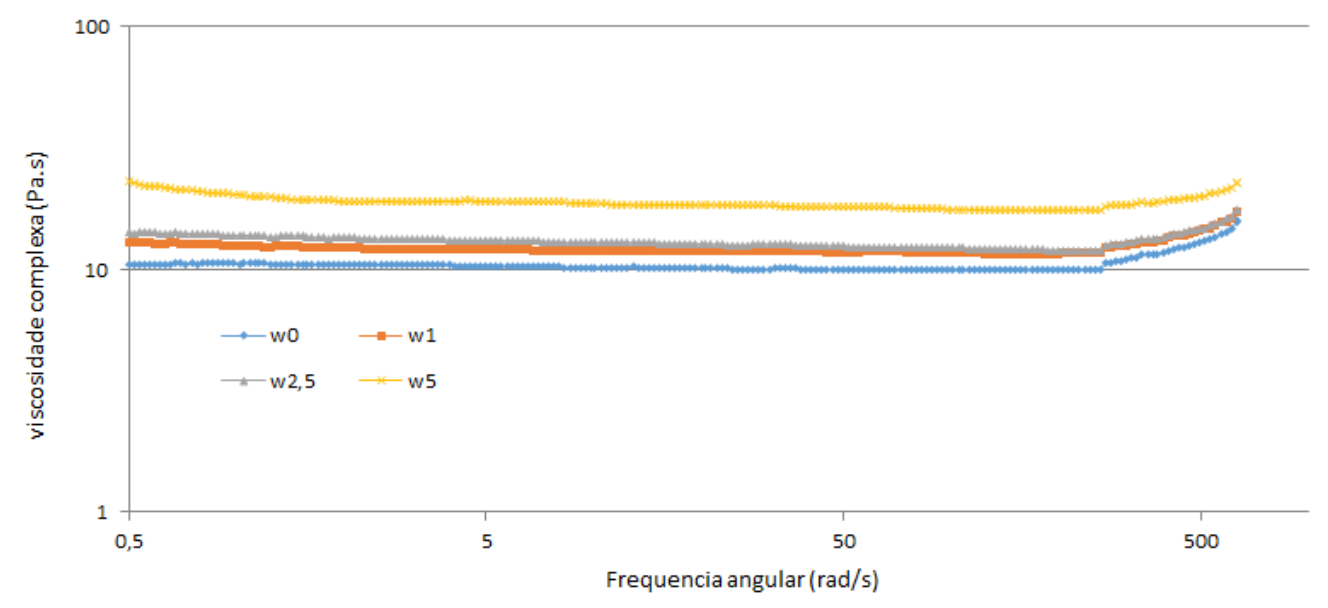

Fonte: Os autores.

\section{CONCLUSÃO}

Foram propostos neste trabalho os principais aspectos envolvidos na modificação da resina epóxi (RE), curada com agente endurecedor poliamida e nesta matriz epoxídica, incorporados nanopartículas inorgânicas de sílica comercial - R200 $\left(\right.$ Aerosil $\left.{ }^{\circledR}\right)$. As nanopartículas sólidas são as responsáveis pela melhor resistência à tração, contração durante a cura, além de aumentarem a resistência térmica e a temperatura de transição vítrea.

A caracterização das amostras dos sistemas epóxi/poliamida, com e sem adição de nanocarga de sílica foram avaliadas por Termogravimetria (TG) e Termogravimetria derivativa (DTG) para o sistema empregando $54 \mathrm{phr}$ de poliamida. Observa-se que os perfis das curvas de DTG são semelhantes, apresentando três eventos principais na faixa de temperatura compreendido entre 362 a $427^{\circ} \mathrm{C}$. Verifica-se que o acréscimo de $1 \%$ de nanocargas de sílica promoveu pequena diminuição dos valores de temperatura 
de degradação do materiallevando a temperatura de $375^{\circ} \mathrm{C}$ (T1) para $362^{\circ} \mathrm{C}$ (T1') para o primeiro evento de degradação, a variação na redução de temperatura foi de $13^{\circ} \mathrm{C}$. No segundo evento a temperatura de degradação de $418^{\circ} \mathrm{C}$ (T2) para $410^{\circ}$ (T2'), a variação na redução de temperatura foi de $8^{\circ} \mathrm{C}$. E no terceiro evento térmico a temperatura de degradação térmica também foi semelhante $427^{\circ} \mathrm{C}$.

A caracterização por DMA, as curvas do módulo de armazenamento (E') para o sistema epóxi/poliamida puro e com adição de carga. Nota-se que a amostra com adição de $1 \%$ de carga apresentou o maior valor de módulo de armazenamento (1543Mpa) e não a amostra $5 \%$ de nanocarga como era esperado. Isso pode ser atribuído a um efeito de dispersão. A matriz com menor teor de nanocarga tem a maior probabilidade de ser melhor distribuída na matriz polimérica.

A Reometria das amostras constatou que pela determinação do regime de viscoelasticidade linear da matriz epoxídica, verificou-se que a adição de $1 \% \mathrm{~m} / \mathrm{m}$ da sílica resultou no aumento da viscosidade da matriz epoxídica como esperado. Este comportamento sugere que a dispersão resultou na maior interação entre a ER e a carga de sílica, promovido pelo alto cisalhamento durante o procedimento de mistura.

\section{AGRADECIMENTOS}

Os autores agradecem à FAPERJ e ao CNPq pelo fomento à pesquisa nas Universidades, bem como a professora Bluma Soares e a equipe do laboratório J112 IMA-UFRJ pela realização das análises de DMA e Reologia.

\section{REFERÊNCIAS}

AHMED, L. et al. Application of polymer nanocomposites in the flame retardancy study. Journal of Loss Prevention in the Process Industries, v. 55, p. 381-391, 2018.

ANADÃO, P.; WIEBECK, H.; VALENZUELA-DÍAZ, F. R. Panorama da pesquisa acadêmica brasileira em nanocompósitos polímero/argila e tendências para o Futuro. Polímeros, v. 21, n. 5, p. 443-452, 2011.

CANEVAROLO, S. V. J; MATOS, J. R.; MACHADO, L. D. B. Técnicas de caracterização de polímeros: Análise térmica, termogravimetria, São Paulo: Artliber, 2007.

CARVALHO, A. P. Desenvolvimento de sílica gel organicamente modificada com líquido iônico (Ormosil) e sua utilização em dispersões de resina. 103 f. 2015. Dissertação (Mestrado em Ciência e Tecnologia de Polímeros) - Universidade Federal do Rio de Janeiro, Rio de Janeiro, 2015.

CHEN,Q. et al. Nanoscale and effective mechanical behavior and fracture of silica nanocomposites. Composites Sci and Tech, v. 68, p. 3137-3144, 2008. 
COELHO C. P. D. Obtenção e caracterização de nanocompósitos de poliestireno e argilas esmectíticas. 107f. 2008. Dissertação (Mestrado em Engenharia de Materiais) - Universidade de São Paulo, 2008.

ESTEVES, A. C.; TIMMOS, A. B.; TRINDADE, T. Nanocompósito de matriz polimérica: estratégias de síntese de materiais híbridos. Química Nova, v. 27, n. 5, p 798-806; 2004.

FERREIRA H. S.; RANGEL M. C. Nanotecnologia: Aspectos gerais e potencial de aplicação em catálise. Química Nova, v. 32, n. 7, 2009.

GAO, D. et al. Preparation of epoxy-acrylate copolymer/nano-silica via Pickering emulsion polymerization and its application as printing binder. Applied Surface Science, v. 435, p. 195-202, 2018.

HAZARIKA, D.; KARAK, N. Nanocomposite of waterborne hyperbranched polyester and clay carbon dot as a robust photocatalyst for environmental remediation. Applied Surface Science., v. 498, p. 1-13, 2019.

KARNATI, S. R.; AGBO, P.; ZHANG, L. Applications of silica nanoparticles in glass/carbon fiber-reinforced epoxy nanocomposite. Composites Communications, v. 17 , p. 32-41, 2020.

KAYA, G. G.; YILMAZ, E.; DEVECI, H. Sustainable nanocomposites of epoxy and silica xerogel synthesized from corn stalk ash: Enhanced thermal and acoustic insulation performance. Composites Part B, v. 150, p. 1-6, 2018.

$\mathrm{LI}, \mathrm{H}$. et al. Synergetic enhancement of mechanical and electrical strength in epoxy/silica nanocomposites via chemically-bonded interface. Composites Science and Technology, v. 167, p. 539-546, 2018.

LUCAS, E. F.; SOARES, B. G.; MONTEIRO, E. E. C. Caracterização de polímeros: Determinação do peso molecular e análise térmica. Rio de Janeiro: E-papers, 2001.

MACIEL, A. P.; LONGO, E.; LEITE, E. R.; Dióxido de estanho nanoestruturado: síntese e crescimento de nanocristais e nanofitas; Química Nova, v. 26, n. 6; 2003.

PAVOSKI, G. et al. Synthesis of polyethylene/silica-silver nanocomposites with antibacterial properties by in situ polymerization. European Polymer Journal, v. 106, p. 92-101, 2018.

REDDY, C.; PATRA, P.; DAS, C. Ethylene-octene copolymer-nanosilica nanocomposites: effects of epoxy resin functionalized nanosilica on morphology, mechanical, dynamic mechanical and thermal properties. Macromolecular Symposia, v. 277, n. 1, p. 119-129, 2009.

REZENDE, M. C.; BOTELHO, E. C. O uso de compósitos estruturais na indústria aeroespacial. Polímeros: Ciência e tecnologia, v. 10, n. 2; 2000.

ROENNER, N. et al. Simultaneous improvements in flammability and mechanical toughening of epoxy resins through nano-silica addition. Fire Safety Journal, v. 91, p. 200-207, 2017.

SALIMIAN, S. et al. Fabrication and evaluation of silica aerogel-epoxy nanocomposites: Fracture and toughening mechanisms. Theoretical and Applied Fracture Mechanics, v. 97, p.156-164, 2018. 
SINGH, N. P.; GUPTA, V. K.; SINGH, A. P. Graphene and carbon nanotube reinforced epoxy nanocomposites: A review; Polymer., v. 180, n. 121724, p. 1-21, 2019. 